\title{
Paroxysmal Sympathetic Hyperactivity
}

\section{Jacqueline Urtecho, $\mathrm{MD}^{1,2}$ \\ ${ }^{1}$ Department of Neurological Surgery, Thomas Jefferson University, Philadelphia, PA 2 Department of Neurology, Thomas Jefferson University, Philadelphia, PA}

It is not uncommon for physicians who treat patients with traumatic brain injuries to see wide fluctuations in the heart rate, respiratory rate and blood pressure. For decades, these fluctuations were thought to be seizures, caused by pressure on the thalamus. They were originally termed Diencephalic Autonomic Seizures by Dr. Wilder Penfield in 1929. He described episodes of lacrimation, hypertension, diaphoresis and agitation. Dr. Penfield's "seizures" were later shown to have no electrographic correlate. Since that time, many names have been used to describe similar episodes: Dysautonomia, Sympathetic Storming, Brainstem Attacks, Autonomic Dysregulation, Paroxsymal Autonomic Instability with Dystonia and Paroxysmal Sympathetic Hyperactivity to name only a few.

Paroxysmal Sympathetic Hyperactivity (PSH) occurs in acquired brain injury and features simultaneous, paroxysmal transient increases in sympathetic and motor activity. ${ }^{1}$ It is most commonly associated with traumatic brain injury. However, it has been documented in many neurologic conditions (Table 1) and an episode can be precipitated by a variety of triggers. ${ }^{2}$ (Table 2 ).

One of the difficulties in recognizing PSH is that many of the symptoms are found in other clinical syndromes. It is a diagnosis of exclusion and the proper workup must be completed before beginning treatment. ${ }^{3}$ The clinical features of PSH include tachycardia, tachypnea, hypertension, fever, diaphoresis and dystonic posturing during the episodes. Table 3 highlights many of the conditions which share similar features to PSH.
Table 1. Neurologic Conditions associated

with Paroxysmal Sympathetic Hyperactivity

Traumatic Brain Injury

Anoxic Brain Injury

Ischemic Stroke

Intracranial Hemorrhage

Aneurysmal Subarachnoid Hemorrhage

Brain Tumor

Encephalitis

Table 2. Triggers Precipitating PSH Attack

Suctioning

Turning

Bathing

Physical Exam

PSH has been described as occurring in three phases. ${ }^{4}$ The first phase occurs immediately after the injury. At this early point in the disease process, there

\section{Table 3.}

\begin{tabular}{|l|l|l|l|l|l|l|l|l|l|l|}
\hline & $\begin{array}{l}\text { Mental } \\
\text { Status }\end{array}$ & T & HR & RR & BP & Pupil Size & Sweating & Agitation & Posturing & CPK \\
\hline $\begin{array}{l}\text { Paroxsymal } \\
\text { Sympathetic } \\
\text { Hyperactivity }\end{array}$ & $\downarrow$ & $\uparrow$ & $\uparrow$ & $\uparrow$ & $\uparrow$ & $\uparrow$ & + & + & $\uparrow$ \\
\hline Malignant Hyperthermia & $\downarrow$ & $\uparrow$ & $\uparrow$ & $\uparrow$ & $\pm \uparrow$ & NA & NA & NA & $+>-$ \\
\hline NMS & $\downarrow$ & $\uparrow$ & $\uparrow$ & $\uparrow$ & $\uparrow / \downarrow$ & NA & + & NA & + \\
\hline Increased ICP & $\downarrow$ & & $\downarrow$ & $\downarrow$ & $\uparrow$ & $\pm \uparrow$ & NA & NA & \pm \\
\hline Central Fever & $\pm \downarrow$ & $\uparrow$ & $\uparrow$ & $\uparrow$ & NA & NA & NA & NA & NA & NA \\
\hline Infection & $\pm \downarrow$ & $\uparrow$ & $\uparrow$ & $\uparrow$ & $\uparrow / \downarrow$ & & \pm & NA & NA & NA \\
\hline $\begin{array}{l}\text { Nonconvulsive } \\
\text { seizures/epilepsy }\end{array}$ & NA & NA & NA & NA & NA & $\pm \uparrow$ & NA & \pm & NA & NA \\
\hline Narcotic Withdrawal & $\pm \downarrow$ & NA & $\uparrow$ & $\uparrow$ & NA & $\uparrow$ & + & NA & NA & NA \\
\hline Autonomic dysreflexia & NA & $\uparrow$ & $\uparrow$ & $\uparrow$ & $\uparrow$ & NA & + & NA & NA & NA \\
\hline
\end{tabular}

Abbreviations: NMS, neuroleptic malignant syndrome; T, temperature; HR, heart rate; RR, respiratory rate; BP, blood pressure; CPK, creatine phosphokinase; up arrow, increased; down arrow, decreased. Adapted from Blackman et al Archives of Neurology 2004;61:321-328 
Table 4. Severity of Clinical Features Assessment Tool

Paroxysmal Sympathetic Hyperactivity - Assessment Measure Clinical Features Scale

\begin{tabular}{|l|l|l|l|l|l|}
\hline & $\mathbf{0}$ & $\mathbf{1}$ & $\mathbf{2}$ & $\mathbf{3}$ & Score \\
\hline Heart Rate & $<100$ & $100-119$ & $120-139$ & $\geq 140$ & \\
\hline $\begin{array}{l}\text { Respiratory } \\
\text { Rate }\end{array}$ & $<18$ & $18-23$ & $24-29$ & $\geq 30$ & \\
\hline $\begin{array}{l}\text { Systolic Blood } \\
\text { Pressure }\end{array}$ & $<240$ & $140-159$ & $160-179$ & $\geq 180$ & \\
\hline Temperature & $<37$ & $37-37.9$ & $38-38.9$ & $\geq 39$ & \\
\hline Sweating & None & Mild & Moderate & Severe & \\
\hline $\begin{array}{l}\text { Posturing } \\
\text { during } \\
\text { episodes }\end{array}$ & None & Mild & Moderate & Severe & \\
\hline \multicolumn{7}{|l|}{} & & & & $\begin{array}{l}\text { CSF } \\
\text { Total }\end{array}$ & \\
\hline
\end{tabular}

\begin{tabular}{|l|l|}
\hline Severity of Clinical Features & CFS Total \\
\hline None & 0 \\
\hline Mild & $1-6$ \\
\hline Moderate & $7-12$ \\
\hline Severe & $\geq 13$ \\
\hline
\end{tabular}

Table 5. Diagnosis Likelihood Tool (DLT)

Score 1 point for each feature present

Clinical features occur simultaneously

Episodes are paroxysmal in nature

Sympathetic over-reactivity to normally non-painful stimuli

Features persist $\geq 3$ consecutive days

Features persist $\geq 2$ weeks post-brain injury

Features persist despite treatment of alternative differential diagnoses

\begin{tabular}{|c|c|}
\hline \multicolumn{2}{|l|}{ Medication administered to decrease sympathetic features } \\
\hline$\geq 2$ episodes daily & \\
\hline Absence of parasympathetic features during episodes & \\
\hline Absence of other presumed cause of features & \\
\hline Antecedent acquired brain injury & \\
\hline
\end{tabular}

\begin{tabular}{|l|l|l|}
\hline \multicolumn{2}{|c|}{ Combined Total Points } & \\
\hline \multicolumn{2}{|c|}{ Clinical Severity Features + Diagnostic Likelihood Tool } & \\
\hline $\begin{array}{l}\text { PSH Diagnostic } \\
\text { Likelihood }\end{array}$ & Unlikely $<8$ & \\
\cline { 2 - 3 } & Possible 8-16 & \\
\cline { 2 - 3 } & Probable $>17$ & \\
\hline
\end{tabular}

(Adapted from Baguley I. et al. Journal of Neurotrauma 2014;31:1515-1520) are no specific signs that distinguish a patient who will go on to develop PSH from those who don't. Phase two begins after the withdrawal of sedation and or paralytics. It is at this point that patients distinguish themselves and either develop typical PSH features (hypertension, hyperthermia, rigidity etc.) or don't. The PSH episodes are sporadic and intense at times and have variable responses to medical management. The duration of this phase is unpredictable. It can last from weeks to months. The third phase was called PSH "burnt out." The patient no longer exhibits all the clinical features and can be left in a spastic or dystonic position with varying degrees of recovery.

In 2014, the Journal of Neurotrauma published a consensus statement aimed at formalizing the nomenclature, including definition and diagnostic criteria. Tables 4 and 5 detail the diagnostic criteria.

\section{PAROXYSMAL SYMPATHETIC HYPERACTIVITY - ASSESSMENT MEASURE}

Management of PSH involves both non-pharmacologic and pharmacologic treatment. Non-pharmacologic management includes decreasing external stimuli, limiting visitation, minimizing exams or noxious stimuli, or grouping activities (turning, suctioning, bathing). Pharmacologic management is aimed at dampening sympathetic outflow or activating parasympathetic system. Most commonly used are benzodiazepines, beta-blockers and opiates. Most medical treatment involved depressing the CNS systems and causes increased sedation. ${ }^{5}$ (Table 6).

Managing the symptoms is important in preventing secondary brain injury. Patients who are not treated are at risk for cerebral edema, intracranial bleeding from malignant hypertension. There is a risk of ischemia due to decreased cerebral oxygenation and neuronal loss due to prolonged sympathetic activation. There are other non-brain injury risks that occur due to prolonged untreated $\mathrm{PSH}$. These include electrolyte abnormalities, dehydration and kidney injury from excessive diaphoresis. Cardiac injury can occur from repetitive significant tachycardia and muscle wasting. Weight loss and malnutrition can occur from increased metabolic demands.

Lastly, it is critical that physicians discuss PSH with the families, as these episodes can be very upsetting and distressful to witness. Explaining what is happening to the patient and how it is being managed can help alleviate this stress. It is also a way to involve the family in monitoring for triggers and timing of episodes. Developing a bedside chart which details triggers, timing, duration of episodes, medications administered and response to treatment is useful in the long-term management. 


\begin{tabular}{|c|c|c|c|c|}
\hline & Symptom & $\begin{array}{l}\text { Receptor Agonist or } \\
\text { Antagonist }\end{array}$ & Medication & Additional \\
\hline First Line & Tachycardia & $\beta 2$-Adrenergic blocker & Propranolol & $\begin{array}{l}\text { - Dampens sympathetic activity; decreases serum catechol- } \\
\text { amines, reduces cardiac workload } \\
\text { - Dosing limited by HR and BP } \\
\text { - Caution in asthmatics }\end{array}$ \\
\hline First Line & Hyperthermia & COX-2 inhibitor & $\begin{array}{l}\text { Acetaminophen } \\
\text { (Po 650-975mg q6hr) } \\
\text { (IV 1gm q6h) }\end{array}$ & - Dosing max 4gm/daily \\
\hline First Line & $\begin{array}{l}\text { Diaphoresis \& } \\
\text { hyperthermia }\end{array}$ & Dopamine agonist & $\begin{array}{l}\text { Bromocriptine } \\
\text { (2.5-5mg q8hr) }\end{array}$ & $\begin{array}{l}\text { - Acts at the hypothalamic level. } \\
\text { - Can increase up to } 30-40 \mathrm{mg} / \text { day }\end{array}$ \\
\hline First Line & Tachypnea & GABA-A Antagonist & $\begin{array}{l}\text { Diazepam } \\
\text { (po } 5 \mathrm{mg} \mathrm{q} 8 \mathrm{hr} \text { and titrate } \\
\text { up) }\end{array}$ & $\begin{array}{l}\text { - No max dose } \\
\text { - Dosing limited based on sedation }\end{array}$ \\
\hline First Line & Pain & Opiate Agonist & $\begin{array}{l}\text { Morphine Sulfate } \\
\text { Fentanyl } \\
\text { Oxycodone }\end{array}$ & $\begin{array}{l}\text { - Start low and titrate to effect } \\
\text { - Dosing varies by agent } \\
\text { - High abuse potential long term }\end{array}$ \\
\hline Second Line & Hyperthermia & Dopamine D2 Antagonist & Chlorpromazine & $\begin{array}{l}\text { - Acts along the hypothalamus } \\
\text { - Good for recurrent hyperthemia } \\
\text { - Should not be used long term } \\
\text { - Risk of extra-pyramidal effects \& liver failure }\end{array}$ \\
\hline Second line & Dystonia & GABA-B agonist & Baclofen & $\begin{array}{l}\text { - Low potential for abuse } \\
\text { - Long term use requires slow wean to avoid withdrawal/ } \\
\text { seizures }\end{array}$ \\
\hline Second Line & Dystonia & $\begin{array}{l}\text { Post-synaptic muscle } \\
\text { relaxant, Inhibits Cat } \\
\text { release intracellularly }\end{array}$ & Dantrolene & $\begin{array}{l}\text { - Caution if other } \mathrm{Ca}+\text { Channel Blockers on board can cause } \\
\text { hyperkalemia and } \\
\text { - Caution if liver disease }\end{array}$ \\
\hline Second Line & Tachycardia & $\alpha 2$ Agonist & Clonidine & - Lowers levels of norepinephrine \\
\hline Second Line & Tachyacrdia & $\beta 1, \beta 2, \alpha 1$ antagonist & Labetalol & - Dosing limited by HR and BP \\
\hline
\end{tabular}

\begin{tabular}{|l|l|l|l|l|l|l|l|l|}
\hline Onset time & Trigger & HR & BP & $\begin{array}{l}\text { Diaphoresis } \\
\text { Y/N }\end{array}$ & ICP (mmHg) & $\begin{array}{l}\text { Dystonia } \\
\text { Y/N }\end{array}$ & $\begin{array}{l}\text { Medications } \\
\text { given }\end{array}$ & $\begin{array}{l}\text { Duration of } \\
\text { episode }\end{array}$ \\
\hline & & & & & & & \\
\hline & & & & & & & & \\
\hline & & & & & & & & \\
\hline & & & & & & & & \\
\hline
\end{tabular}

\section{REFERENCES}

1. Baguley IJ et al. Journal of Neurotauma 2014;31:1515-1520

2. Baguley IJ et al. Semin Neurol 2008;28:716-25
3. Blackman et al Archives of Neurology 2004; 61:321-328

4. Baguley IJ et al Journal of Neurosurgical Psychiatry 1999;67:39-43

5. Lemke et al. Crit Care Nurse 2007;27:30-37 